EESTI NSV TEADUSTE AKADEEMIA TOIMETISED. 29. KÖIDE FUOSIKA * MATEMAATIKA. 1980, NR. 3

ИЗВЕСТИЯ АКАДЕМИИ НАУК ЭСТОНСКОЙ ССР. ТОМ 29 ФИЗИКА * МАТЕМАТИКА. 1980. № 3

Л. ТЕМКИН

удК $\quad 517.954: 517.968$

\title{
О ПОСТРОЕНИИ ПЕРИОДИЧЕСКИХ РЕШЕНИИ УРАВНЕНИЯ ПАРАБОЛИЧЕСКОГО ТИПА
}

\author{
(Представил И. Эпик)
}

Работа посвящена построению решений уравнения параболического типа в случае, когда источники действуют периодически по времени. Эти источники распределены как внутри области, где ищется решение задачи, так и на границе. Предлагается способ приближенного построения решения и дается обоснование этого способа. Қраевые задачи для системы уравнений эллиптического типа, получающиеся после разложения решения в тригонометрический ряд по времени, заменяются эквивалентной системой интегральных уравнений Фредгольма второго рода на границе области. Система интегральных уравнений исследуется методами теории потенциала.

На практике подобные задачи с периодически действующими источниками возникают, например, при исследовании теплового режима электрических машин и электронного оборудования.

Задача о тепловых волнах восходит к исследованиям Фурье о колебаниях температурного поля Земли [1]. Ряд задач о тепловых волнах рассмотрен в $\left.{ }^{2}\right]$.

\section{1. Постановка задачи}

Пусть область $\Omega \subset \mathbf{R}^{m}$ ( $m \geqslant 2$ ) ограничена кусочно-гладкой поверхностью $S=\partial \Omega$. Будем искать решение $u(X, t)$ задачи без начального условия для уравнения параболического типа

$$
\begin{gathered}
c(X) \frac{\partial u(X, t)}{\partial t}=-\Omega u(X, t)+q(X, t), \quad \Omega u=-\operatorname{div}(\lambda \operatorname{grad} u)+\gamma u, \\
q(X, t+T)=q(X, t), \quad X=\left(x_{1}, x_{2}, \ldots, x_{m}\right) \in \Omega
\end{gathered}
$$

при граничном условии

$$
\lambda(X) \frac{\partial u(X, t)}{\partial n}+h(X) u(X, t)=f(X, t), \quad f(X, t+T)=f(X, t), \quad X \in S .
$$

Здесь $n$-внешняя нормаль к поверхности $S, c(X) \in C(\bar{\Omega}), \lambda(X) \in C^{1}(\bar{\Omega})$, $\gamma(X) \in C(\bar{\Omega}), h(X) \in C(S), c(X)>0, \lambda(X)>0, \gamma(X) \geqslant 0, h(X) \geqslant 0$.

Нас будет интересовать поведение решения при достаточно больших значениях времени $t$ (или, что то же самое, если начальный момент времени $\left.t_{0} \rightarrow-\infty\right)$, қкогда влиянием начального условия уже можно пренебречь. Из общего представления решения начально-краевой задачи с помощью функции Грина и асимптотического представления 
функции Грина при $t_{0} \rightarrow-\infty$ легко убедиться в том, что начальное значение функции $u$ добавляет в асимптотическое представление решения слагаемое, экспоненциально убывающее по $t_{0}$, и постоянное слагаемое при условии

$$
\gamma(X) \equiv 0 \wedge h(X) \equiv 0 .
$$

Следовательно, решение задачи (1.1)-(1.2) определено с точностью до экспоненциально убывающего по $t$ (или экспоненциально убывающего и постоянного) слагаемого.

Будем искать периодическое решение, т. е. будем рассматривать задачу $(1.1)-(1.2)$ на отрезке времени $[0, T]$ при условиях

$$
\begin{gathered}
u(X, t+T)=u(X, t), \\
\gamma(X) \neq 0 \vee h(X) \neq 0 .
\end{gathered}
$$

Далее будем называть эту задачу задачей (A). Из дальнейшего будет видно, что и при условии (1.3) для фактического построения решения достаточно рассматривать краевую задачу только на отрезке $[0, T]$. Однако в этом случае, вообще говоря, не существует решения задачи с условием (1.4), а можно гарантировать существование и (с точностью до аддитивной постоянной) единственность решения задачи $(1.1)-(1.2)$, удовлетворяющего условию периодичности

$$
\frac{\partial u(X, t+T)}{\partial t}=\frac{\partial u(X, t)}{\partial t} .
$$

Задачу (1.1)-(1.2), (1.6) при условии (1.3) будем далее называть задачей (В).

Периодические по времени $t$ с периодом $T$ функции $q$ и $f$ будем считать заданными в виде рядов Фурье

$q(X, t)=a_{0}(X) / 2+\sum_{k=1}^{\infty}\left[a_{k}(X) \cos k \omega t+b_{k}(X) \sin k \omega t\right], \quad \omega=2 \pi / T, \quad X \in \Omega$,

$$
f(X, t)=\alpha_{0}(X) / 2+\sum_{k=1}^{\infty}\left[\alpha_{k}(X) \cos k \omega t+\beta_{k}(X) \sin k \omega t\right], \quad X \in S,
$$

где коэффициенты Фурье $a_{k}, b_{k} \in H^{v}(\Omega) \cap C(\bar{\Omega})$ и $\alpha_{k}, \beta_{k} \in C(S), H^{v}-$ класс функций, удовлетворяющих условию Гельдера с показателем $v>0$.

Будем искать решения задач (А) и (В) в виде

$$
u(X, t)=u_{-1}(X) t / T+u_{0}(X) / 2+\sum_{k=1}^{\infty}\left[u_{k}(X) \cos k_{\omega} t+v_{k}(X) \sin k_{\omega} t\right] .
$$

Как будет показано ниже, $u_{-1}(X) \not \equiv 0$ только в. случае задачи (В), если суммарный поток за период $T$

$$
\begin{aligned}
Q & =\int_{0}^{T} \int_{\Omega} q(X, t) d \Omega d t+\int_{0}^{T} \int_{\mathrm{S}} f(X, t) d S d t= \\
& =T\left[\int_{\Omega} a_{0}(X) d \Omega+\int_{S} \alpha_{0}(X) d S\right] / 2 \neq 0 .
\end{aligned}
$$

В этом случае, как и в $\left[{ }^{3,4}\right]$, представление решения содержит неограниченное при больших $t$ слагаемое. 


\section{2. Эллиптические краевые задачи}

Подставив разложения (1.7) - (1.9) в уравнение (1.1) и граничное условие (1.2), получим последовательность эллиптических краевых задач относительно коэффициентов Фурье

$$
\begin{array}{ll}
\Omega u_{-1}(X)=0, & X \in \Omega ; \quad \lambda(X) \frac{\partial u_{-1}(X)}{\partial n}+h(X) u_{-1}(X)=0, \quad X \in S ; \\
& 2 c(X) u_{-1}(X) / T=-\Omega u_{0}(X)+a_{0}(X), \quad X \in \Omega ; \\
& \lambda(X) \frac{\partial u_{0}(X)}{\partial n}+h(X) u_{0}(X)=\alpha_{0}(X), \quad X \in S ; \\
& k \omega c(X) v_{k}(X)=-\Omega u_{k}(X)+a_{k}(X), \quad X \in \Omega ; \\
& k \omega c(X) u_{k}(X)=\Omega v_{k}(X)-b_{k}(X), \quad X \in \Omega ; \\
\lambda(X) \frac{\partial u_{k}(X)}{\partial n}+h(X) u_{k}(X)=\alpha_{k}(X), & \\
\lambda(X) \frac{\partial v_{k}(X)}{\partial n}+h(X) v_{k}(X)=\beta_{k}(X), \quad X \in S,
\end{array}
$$

где $k=1,2, \ldots$.

Если выполняется условие (1.5), то краевые задачи (2.1) и (2.2)(2.3) имеют единственное решение. Из (2.1) поэтому следует, что $u_{-1}(X) \equiv 0$. Если же выполняется (1.3), то из (2.1) имеем $u_{-1}=$ const; эта константа определяется из необходимого и достаточного условия разрешимости краевой задачи $(2.2)-(2.3)$

$$
u_{-1}=T\left[\int_{\Omega} a_{0}(X) d \Omega+\int_{S} \alpha_{0}(X) d S\right] / 2 \int_{\Omega} c(X) d \Omega=Q / \int_{\Omega} c(X) d \Omega .
$$

Решение задачи $(2.2)-(2.3)$ при условии (1.3) определяется с точностью до аддитивной постоянной.

Нетрудно показать, что при каждом $k=1,2, \ldots$ краевая задача $(2.4)-(2.6)$ для системы эллиптических уравнений имеет не более одного решения. Действительно, рассмотрим соответствующую однородную задачу. Применяя стандартное рассуждение с использованием формулы Грина, получим

$$
\begin{gathered}
k \omega \int_{\Omega} c\left[u_{k}^{2}+v_{k}^{2}\right] d \Omega=\int_{\Omega}\left[u_{k} \Omega v_{k}-v_{k} \Omega u_{k}\right] d \Omega= \\
=\int_{S} \lambda\left[v_{k} \frac{\partial u_{k}}{\partial n}-u_{k} \frac{\partial v_{k}}{\partial n}\right] d S=-\int_{S} h\left[v_{k} u_{k}-u_{k} v_{k}\right] d S \equiv 0,
\end{gathered}
$$

откуда вытекает, что $u_{k}(X)=v_{k}(X) \equiv 0$. В силу линейности отсюда сразу следует единственность решения неоднородной краевой задачи $(2.4)-(2.6)$.

\section{3. Интегральные уравнения}

Запишем задачу (2.4)-(2.6) в виде

$$
\begin{gathered}
\mathfrak{M}_{k}(X)=F_{k}(X), \quad F_{k}=a_{k}+i b_{k}, \quad w_{k}=u_{k}+i v_{k}, \quad X \in \Omega, \\
\lambda(X) \frac{\partial w_{k}(X)}{\partial n}+h(X) w_{k}(X)=\Phi_{k}(X), \quad \Phi_{k}=\alpha_{k}+i \beta_{k}, \quad X \in S,
\end{gathered}
$$




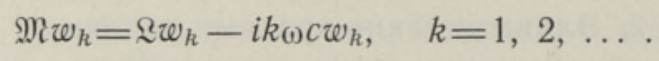

Проверим, что для комплекснозначного оператора $\mathfrak{M}$ выполняется следующий принцип максимума. Пусть ш- решение однородного уравнения

$$
\mathfrak{M} w(Y)=0
$$

в некоторой области $\Omega$. Тогда $|w|$ не может иметь максимума внутри этой области.

Действительно, пусть $|w|$ имеет максимум в некоторой точке $P \in \Omega$. По условию максимума в этой точке должно быть

$$
\operatorname{div}\left(\lambda \operatorname{grad}\left(|w|^{2}\right)\right)<0 \text {. }
$$

С другой стороны, в силу уравнения (3.4), после несложных преобразований получим

$$
\operatorname{div}\left(\lambda \operatorname{grad}\left(|w|^{2}\right)\right)=2 \lambda|\operatorname{grad} w|^{2}+2 \gamma|w|^{2} \geqslant 0
$$

(так как $\lambda>0, \gamma \geqslant 0$ ). Полученное противоречие доказывает утверждение.

Пусть $H(X, Y)$ - фундаментальное решение уравнения (3.4) в области $\Omega$, т. е. функция, являющаяся решением уравнения (3.4) по координатам точки $Y$ при $Y \neq X$ и допускающая представление

$$
H(X, Y)=\left\{\begin{array}{l}
\frac{r^{2-m}(X, Y)}{(m-2) \omega_{m} \lambda(X)}+\varphi_{1}(X, Y)+i \varphi_{2}(X, Y), \omega_{m}=\frac{2 \pi^{m / 2}}{\Gamma(m / 2)}, m \geqslant 3, \\
\frac{1}{2 \pi \lambda(X)} \ln \frac{2 D}{r(X, Y)}+\varphi_{1}(X, Y)+i \varphi_{2}(X, Y), \quad m=2,
\end{array}\right.
$$

где $r(X, Y)$ - расстояние между точками $X$ и $Y, \omega_{m}-$ площадь $(m-1)$-мерной сферы единичного радиуса, $D$ - диаметр области $\Omega$, функции $\varphi_{1}$ и $\varphi_{2}$ удовлетворяют оценкам вида [ [ $\left.{ }^{5}\right]$

$$
\begin{gathered}
\varphi=O\left(r^{v+2-m}\right), \quad \frac{\partial \varphi}{\partial y_{i}}=O\left(r^{v+1-m}\right), \frac{\partial^{2} \varphi}{\partial y_{i} \partial y_{j}}=O\left(r^{v-m}\right) ; \quad v>0 ; \\
i, j=1, \ldots, m .
\end{gathered}
$$

Стандартным образом, вырезая малую окрестность точки $X \in S$, используя формулу Грина и стягивая эту окрестность к точке $X$, получим, что для оператора $\mathfrak{M}$ справедлива формула

$$
\begin{gathered}
p(X) w(X)=\int_{S} \lambda(Y) H(X, Y) \frac{\partial w(Y)}{\partial n} d S_{Y}-\int_{S} \lambda(Y) \frac{\partial H(X, Y)}{\partial n_{Y}} w(Y) d S_{Y}+ \\
+\int_{\Omega} H(X, Y) \mathfrak{w}(Y) d \Omega_{Y}, \quad X \in S
\end{gathered}
$$

Если $X \in S$ и $S$ имеет в точке $X$ касательную гиперплоскость, то $p(X)=1 / 2$, в противном случае $p(X)$ равно величине соответствующего телесного угла, отнесенного к $\omega_{m}$. Если же $X \in \Omega$, то $p(X)=1$.

В дальнейшем будем использовать главное фундаментальное решение $G$ уравнения (3.4), т. е. фундаментальное решение, определенное во всем пространстве $\mathbf{R}^{m}$, причем существуют такие постоянные $a>0$ и $R>0$, что при $r>R$ 


$$
G=O\left(e^{-a r}\right), \quad \frac{\partial G}{\partial y_{i}}=O\left(e^{-a r}\right) .
$$

Функцию $\lambda$ считаем продолженной гладким образом, а функции $c$ и $\gamma$ - непрерывным образом на все пространство $\mathbf{R}^{m}$.

Доказательство существования главного фундаментального решения проводится методом Жиро аналогично случаю вещественного оператора [5], причем такое доказательство содержит и метод построения функции $G(X, Y)$.

Заметим, что главное фундаментальное решение является симметричной функцией точек $X$ и $Y$. Действительно, применим формулу Грина к функциям $G(Y, Z)$ и $G(X, Z)$, рассматриваемым как функции точки $Z$ в области $\Omega_{R}=D_{R} \backslash E_{X} \backslash E_{Y}$, где $E_{X}$ и $E_{Y}$ - шары радиуса $\varepsilon$ с центрами в точках $X$ и $Y, \bar{E}_{X} \cap \bar{E}_{Y}=\Lambda$ ( $\Lambda$ пустое множество), $\bar{E}_{X} \cup \bar{E}_{Y} \subset D_{R}, D_{R}$ - шар радиуса $R$. В силу уравнения (3.4) получим

$$
\begin{gathered}
0 \equiv \int_{\Omega_{R}}\left[G(X, Z) \mathfrak{M}_{Z} G(Y, Z)-G(Y, Z) \mathfrak{M}_{Z} G(X, Z)\right] d \Omega_{Z}= \\
=-\int_{\Sigma_{R}} \lambda(Z)\left[G(X, Z) \frac{\partial G(Y, Z)}{\partial n_{Z}}-G(Y, Z) \frac{\partial G(X, Z)}{\partial n_{Z}}\right] d S_{Z},
\end{gathered}
$$

где $\Sigma_{R}=S_{R} \cup \Gamma_{X} \cup \Gamma_{Y}, S_{R}=\bar{D}_{R} \backslash D_{R}, \Gamma_{X}=\bar{E}_{X} \backslash E_{X}, \quad \Gamma_{Y}=\bar{E}_{Y} \backslash E_{Y}$.

Переходя в правой части (3.9) к пределу при $R \rightarrow \infty$ и $\varepsilon \rightarrow 0$, с использованием асимптотических представлений (3.8) и (3.5) получим, что интеграл по $S_{R}$ стремится к нулю, а интегралы по $\Gamma_{X}$ и $\Gamma_{Y}-$ coответственно к $-G(Y, X)$ и $G(X, Y)$. Из (3.9) поэтому следует, что

$$
G(Y, X)=G(X, Y) \text {. }
$$

Нетрудно проверить, что если $\Omega$ - оператор с постоянными коэффициентами ( $c=$ const, $\lambda=$ const, $\gamma=$ const), главное фундаментальное решение имеет вид

$$
\begin{gathered}
G(X, Y)= \\
=\left\{\begin{array}{l}
\frac{1}{(2 \pi)^{p} \lambda}\left(\frac{x}{r}\right)^{p-1} K_{p-1}(\varkappa r) ; p=\frac{m}{2}, m=2,4,6, \ldots, \\
\frac{r^{-q-1} e^{-x r}}{(m-2) \omega_{m} \lambda} \sum_{i=0}^{q} \frac{x^{i}}{i ! r^{q-i}} ; q=\frac{m-3}{2}, m=3,5,7, \ldots .
\end{array}\right.
\end{gathered}
$$

Здесь $K_{p-1}(x r)$ - функция Макдональда, $r=r(X, Y)$,

$$
x=\left[\frac{\left(k^{2} \omega^{2} c^{2}+\gamma^{2}\right)^{1 / 2}+\gamma}{2 \lambda}\right]^{1 / 2}-i\left[\frac{\left(k^{2} \omega^{2} c^{2}+\gamma^{2}\right)^{1 / 2}-\gamma}{2 \lambda}\right]^{1 / 2} .
$$

Указанная функция $G(X, Y)$ удовлетворяет условиям (3.5), (3.6) и (3.8).

Далее поверхность $S$ будем считать достаточно гладкой. Используя уравнение (3.1) и граничное условие (3.2), из формулы (3.7) получим уравнение на поверхности $S$ относительно комплекснозначной функции $w_{k}(X)$

$$
\frac{1}{2} w_{k}(X)+\int_{S}\left[h(Y) G(X, Y)+\lambda(Y) \frac{\partial G(X, Y)}{\partial n_{Y}}\right] w_{k}(Y) d S_{Y}=
$$




$$
=\int_{S} G(X, Y) \Phi_{k}(Y) d S_{Y}+\int_{\Omega} G(X, Y) F_{k}(Y) d \Omega_{Y}, \quad X \in S,
$$

или, что то же самое, систему интегральных уравнений относительно функций $u_{k}(X)$ и $v_{k}(X)$

$$
\begin{aligned}
& \frac{1}{2} u_{k}+\int_{S}\left(h G_{1}+\lambda \frac{\partial G_{1}}{\partial n}\right) u_{k} d S_{Y}-\int_{S}\left(h G_{2}+\lambda \frac{\partial G_{2}}{\partial n}\right) v_{k} d S_{Y}= \\
& =\int_{S}\left(G_{1} \alpha_{k}-G_{2} \beta_{k}\right) d S_{Y}+\int_{\Omega}\left(G_{1} a_{k}-G_{2} b_{k}\right) d \Omega_{Y}, \quad X \in S ; \\
& \frac{1}{2} v_{k}+\int_{S}\left(h G_{2}+\lambda \frac{\partial G_{2}}{\partial n}\right) u_{k} d S_{Y}+\int_{S}\left(h G_{1}+\lambda \frac{\partial G_{1}}{\partial n}\right) v_{k} d S_{Y}= \\
& =\int_{S}\left(G_{1} \beta_{k}+G_{2} \alpha_{k}\right) d S_{Y}+\int_{\Omega}\left(G_{1} b_{k}+G_{2} a_{k}\right) d \Omega_{Y}, \quad X \in S .
\end{aligned}
$$

Здесь $G_{1}=\operatorname{Re} G, G_{2}=\operatorname{Im} G$.

Из приведенного выше вывода системы интегральных уравнений (3.13)-(3.14) следует, что если решение $\left(u_{k}, v_{k}\right)$ краевой задачи (2.4)-(2.6) таково, что $u_{k}, v_{k} \in C^{1}(\bar{\Omega}) \cap C^{2}(\Omega)$ (условия применимости формулы Грина), то его след на границе области удовлетворяет системе уравнений (3.13)-(3.14). Докажем обратное утверждение.

Т е орем а. 1. Пусть поверхность $S$ в окрестности каждой своей точки в некоторой локальной декартовой системе координат может быть задана явной функцией $\xi_{m}=\zeta\left(\xi_{1}, \ldots, \xi_{m-1}\right)$, имеющей непрерывные вторые частные производные. Будем предполагать, что $\lambda \in C^{1}\left(\mathbf{R}^{m}\right), h \in C(S), c \in C\left(R^{m}\right), \quad \gamma \in C\left(R^{m}\right), \quad c>0, \quad \lambda>0 ; \quad a_{k}, b_{k} \in$ $\in H^{v}(\Omega) \cap C(\bar{\Omega})$ и $\alpha_{k}, \beta_{k} \in C(S)$ nрu $k=1,2, \ldots$.

Тогда функция

$$
\begin{aligned}
& W(X)=-\int_{S}\left[h(Y) G(X, Y)+\lambda(Y) \frac{\partial G(X, Y)}{\partial n_{Y}}\right] w_{k}(Y) d S_{\mathbf{Y}}+ \\
& +\int_{S} G(X, Y) \Phi_{k}(Y) d S_{Y}+\int_{\Omega} G(X, Y) F_{k}(Y) d \Omega_{Y} \quad(X \in \Omega)
\end{aligned}
$$

является решением задачи (3.1)-(3.2), если $w_{k} \in L_{2}(S)$ - решение уравнения (3.12), а $G(X, Y)$ - главное фундаментальное решение уравнения (3.4). Переходы (3.1) U(3.2)↔(3.12) являются взаимно-обратными.

Д оказ ательство. При сделанных выше предположениях правая часть в уравнении (3.12) непрерывна в силу свойств обобщенных потенциалов, рассмотренных в [5]. Следовательно, решение этого уравнения со слабой особенностью $w_{k} \in C(S)$.

В силу $(3,10)$ главное фундаментальное решение $G(X, Y)$ будет решением уравнения (3.4) как по координатам точки $Y$, так и по координатам точки $X$. Поэтому из (3.15) в силу свойств обобщенных потенциалов следует, что $W(X)$ удовлетворяет уравнению (3.1).

С учетом скачка обобщенного потенциала двойного слоя получаем сразу 


$$
\lim _{X \rightarrow X_{0}} W(X)=w_{k}\left(X_{0}\right), \quad X \in \Omega, \quad X_{0} \in S .
$$

Обозначим через $W_{e}(X)$ функцию, определяемую правой частью формулы (3.15) при $X \in \Omega_{e}=\mathbf{R}^{m} \backslash \bar{\Omega}$. Функция $W_{e}(X)$ удовлетворяет уравнению (3.4) и $\left|W_{e}\right| \rightarrow 0$ при. $|X| \rightarrow \infty$. Кроме того, с учетом скачка обобщенного потенциала двойного слоя в силу уравнения (3.12) имеем

$$
\lim _{X \rightarrow X_{0}} W_{e}(X)=0, \quad X \in \Omega_{e}, \quad X_{0} \in S .
$$

Поскольку для уравнения (3.1) имеет место единственность решения внешней задачи Дирихле, что сразу следует из доказанного в начале этого раздела принципа максимума, то

$$
W_{e}(X) \equiv 0, \quad X \in \Omega_{e} .
$$

Продифференцируем функцию $W_{e}(X)$ по нормали к поверхности $S$ и перейдем к пределу извне. С учетом скачка нормальной производной обобщенных потенциалов простого слоя получим

$$
\begin{gathered}
0 \equiv \lim _{X \rightarrow X_{0}} \frac{\partial W_{e}(X)}{\partial n}=\frac{h\left(X_{0}\right)}{2 \lambda\left(X_{0}\right)} w_{k}\left(X_{0}\right)-\int_{S} h(Y) G\left(X_{0}, Y\right) w_{k}(Y) d S_{Y}- \\
\quad-\lim _{X \rightarrow X_{0}} \frac{\partial}{\partial n_{X}} \int_{S} \lambda(Y) \frac{\partial G(X, Y)}{\partial n_{Y}} w_{k}(Y) d S_{Y}-\frac{\Phi_{k}\left(X_{0}\right)}{2 \lambda\left(X_{0}\right)}+\quad \\
+\int_{S} G\left(X_{0}, Y\right) \Phi_{k}(Y) d S_{Y}+\int_{\Omega} G\left(X_{0}, Y\right) F_{k}(Y) d \Omega_{Y}, \quad X_{0} \in S, \quad X \in \Omega_{e} .
\end{gathered}
$$

Отсюда следует, что существует предел нормальной производной потенциала двойного слоя извне. Для обобщенного потенциала двойного слоя имеет место аналог теоремы Ляпунова о нормальной производной [5]. Следовательно, существует предел изнутри, равный пределу извне. С использованием формулы (3.17) получим

$$
\lim _{x \rightarrow X_{0}} \frac{\partial W}{\partial n}=-\frac{h\left(X_{0}\right) w_{k}\left(X_{0}\right)}{2 \lambda\left(X_{0}\right)}-\int_{S} h(Y) G\left(X_{0}, Y\right) w_{k}(Y) d S_{Y}-
$$

$-\lim _{\boldsymbol{X} \rightarrow X_{0}} \frac{\partial}{\partial n_{X}} \int_{S} \lambda(Y) \frac{\partial G(X, Y)}{\partial n_{Y}} w_{k}(Y) d S_{Y}+\frac{\Phi_{k}\left(X_{0}\right)}{2 \lambda\left(X_{0}\right)}+\int_{S} G\left(X_{0}, Y\right) \Phi_{k}(Y) d S_{Y}+$

$+\int_{\Omega} G\left(X_{0}, Y\right) F_{k}(Y) d \Omega_{Y}=-\frac{h\left(X_{0}\right) w_{k}\left(X_{0}\right)}{\lambda\left(X_{0}\right)}+\frac{\Phi_{k}\left(X_{0}\right)}{\lambda\left(X_{0}\right)}, \quad X X_{0} \in S, \quad X \in \Omega$.

Принимая во внимание $(3.16)$, из (3.18) получим, что функция $W(X)$ удовлетворяет граничному условию (3.2).

Поскольку функция $W(X)$ является классическим решением краевой задачи (3.1)-(3.2), она удовлетворяет условиям, необходимым для применения формулы Грина. Отсюда следует последнее утверждение теоремы.

Из теоремы 1 сразу вытекает существование и единственность в $L_{2}(S)$ решения интегрального уравнения (3.12), а следовательно, и существование решения краевой задачи (3.1)-(3.2).

Действительно, пусть однородное уравнение, соответствующее (3.12), имеет нетривиальное решение $w^{0} \in L_{2}(S)$. Тогда, как было отмечено 
в доказательстве теоремы $1, w^{0} \in C(S)$. По теореме 1 соответствующая функция $W^{0}(X)$ будет решением краевой задачи $(3.1)-(3.2)$ с нулевыми правыми частями и, как было показано в разделе $2, W^{0}(X) \equiv 0$. Отсюда следует, что $w^{0}=W^{0} \mid s \equiv 0$. Так как оператор интегрального уравнения (3.12) вполне непрерывен в $L_{2}(S)$, из теоремы Фредгольма следует существование решения этого уравнения при любых правых частях из $L_{2}(S)$.

Используя свойства обобщенных потенциалов, нетрудно показать, что для существования решения уравнения (3.12) достаточно потребовать, чтобы $a_{k}, b_{k} \in L_{2}(\Omega)$, а $\alpha_{k}, \beta_{k} \in L_{2}(S)$. Теорема 1 справедлива и в этом случае, причем $W \in W_{2}^{2}(\Omega)$ будет обобщенным решением задачи (3.1) - (3.2), а краевое условие (3.2) удовлетворяется почти всюду.

Заметим, что 'в этом разделе не иопользовалось ограничение $h \geqslant 0$.

\section{4. Нулевая гармоника}

Рассмотрим теперь краевую задачу (2.2)-(2.3). При $\gamma \geqslant 0, h \geqslant 0$ существование и единственность (при условии (1.3) - с точностью до аддитивной постоянной) решения этой задачи, а также существование и единственность главного фундаментального решения $G_{0}(X, Y)$ для оператора 2 доказано в [5], причем для построения этого фундаментального решения необходимо продолжить функцию $\gamma(X)$ на все пространство так, чтобы было $\gamma(X)>0$ вне некоторой ограниченной области. Функция $G_{0}(X, Y)$ удовлетворяет условию симметрии $G_{0}(X, Y)=$ $=G_{0}(Y, X)$.

Используя главное фундаментальное решение, получим интегральное уравнение относительно $u_{0} \mid \mathrm{s}$

$$
\begin{gathered}
\frac{1}{2} u_{0}(X)+\int_{S}\left[h(Y) G_{0}(X, Y)+\lambda(Y) \frac{\partial G_{0}(X, Y)}{\partial n_{Y}}\right] u_{0}(Y) d S_{Y}=F(X), \\
X \in S \\
F(X)=\int_{S} G_{0}(X, Y) \alpha_{0}(Y) d S_{Y}+2 \int_{\Omega} G_{0}(X, Y)\left[\frac{a_{0}(Y)}{2}-\frac{c(Y) u_{-1}}{T}\right] d \Omega_{Y} .
\end{gathered}
$$

Очевидно, что если $u_{0}(X)$ - решение краевой задачи $(2.2)-(2.3)$, то $u_{0} \mid s$ удовлетворяет интегральному уравнению (4.1). Совершенно аналогично теореме 1 с очевидными упрощениями, связанными с вещественностью оператора задачи и неизвестной функции, доказывается следующая

Т е о р е м а 2. Пусть поверхность $S$, а такюе функции $\lambda, c, \gamma u h$ удовлетворяют тем же условиям, что и в теореме 1 , функции $a_{0}, \alpha_{0}-$ тем же условиям, что и $a_{k}, \alpha_{k}$. Функция $\gamma$, кроме того, удовлетворяет условиям, указанным в начале этого раздела, $u_{0} \in L_{2}(\Omega)$ - решение интегрального уравнения (4.1). Тогда функция

$$
U(X)=-\int_{S}\left[h(Y) G_{0}(X, Y)+\lambda(Y) \frac{\partial G_{0}(X, Y)}{\partial n_{Y}}\right] u_{0}(Y) d S_{Y}+F(X) \quad(X \in \Omega)
$$

является решением краевой задачи (2.2)-(2.3).

При условии (1.5) из теоремы 2 и единственности решения задачи (2.2) - (2.3) вытекает единственность решения интегрального уравнения (4.1). Если же имеет место (1.3), уравнение (4.1), как и краевая задача 
(2.2) - (2.3), имеет решение, определенное с точностью до аддитивной постоянной. Поскольку однородное уравнение, соответствующее (4.1), имеет нетривиальное решение (равное постоянной), возникают трудности при построении приближенного решения уравнения (4.1), так как конечномерная аппроксимация интегрального оператора приводит к матрице с нулевым определителем. Это замечание (при условии (1.3) ) справедливо и для краевой задачи (2.2) - (2.3), если для ее решения применять сеточные методы. Чтобы избежать отмеченной трудности, решение задачи следует искать в подпространстве $L_{2}(S) \ominus\{1\}$, т. е. среди функций, удовлетворяющих условию ортогональности

$$
\int_{S} u(X) d S=0 .
$$

Применяя к обеим частям (4.1) операцию ортогонального проектирования, с учетом (4.4) получим

$$
\begin{gathered}
\frac{1}{2} u_{0}(X)+\int_{S} \lambda(Y)\left[\frac{\partial G_{0}(X, Y)}{\partial n_{Y}}-\frac{1}{\operatorname{mes} S} \int_{S} \frac{\partial G_{0}(Z, Y)}{\partial n_{Y}} d S_{Z}\right] u_{0}(Y) d S_{Y}= \\
=F(X)-\frac{1}{\operatorname{mes} S} \int_{S} F(X) d S, \quad X \in S .
\end{gathered}
$$

Т еорем а 3. Уравнение (4.5) имеет единственное решение.

Доказ а тельство опирается на следующий простой факт.

Пусть $\varphi_{0}$ - единственное, с точностью до числового множителя, нетривиальное решение однородного уравнения $\varphi=\mathfrak{A} \varphi$, где $\mathfrak{A}$ - линейный вполне непрерывный оператор в некотором гильбертовом пространстве $\mathfrak{g} ; \sigma_{0}$ - нетривиальное решение однородного сопряженного уравнения $\sigma=\mathfrak{U}^{*} \sigma$. Тогда, для того чтобы однородное уравнение

$$
\varphi=P \mathfrak{A} \varphi, \quad P: \mathscr{G} \rightarrow \mathscr{S} \ominus\left\{\varphi_{0}\right\}
$$

( $P$ - оператор ортогонального проектирования) не имело нетривиального решения, необходимо и достаточно, чтобы выполнялось условие

$$
\left(\varphi_{0}, \sigma_{0}\right) \neq 0 \text {. }
$$

Действительно, пусть $\varphi_{1} \not \equiv 0-$ решение уравнения (4.6); тогда $P\left(\varphi_{1}-\mathfrak{A} \varphi_{1}\right)=0$. Следовательно, $\varphi_{1}=\mathfrak{A} \varphi_{1}+C \varphi_{0}, C=$ const $\neq 0$ и по теореме Фредгольма $\left(\varphi_{0}, \sigma_{0}\right)=0$. И обратно, при условии $\left(\varphi_{0}, \sigma_{0}\right)=0$ уравнение $\varphi=\mathfrak{N} \varphi+\varphi_{0}$ имеет решение, определенное с точностью до слагаемого $\bar{C}$ р. Произвольную постоянную $\bar{C}$ можно выбрать так, чтобы это решение удовлетворяло условию $P \varphi_{1}=\varphi_{1}$; тогда $\varphi_{1}-$ нетривиальное решение уравнения (4.6).

В нашем случае $\varphi_{0}=$ const, и условие (4.7) принимает вид

$$
\int_{S} \sigma_{0}(X) d S \neq 0,
$$

где $\sigma_{0}(X)$ - решение сопряженного к (4.1) однородного уравнения (при $h \equiv 0$ )

$$
\frac{1}{2} \sigma(X)+\lambda(X) \int_{S} \frac{\partial G_{0}(X, Y)}{\partial n_{X}} \sigma(Y) d S_{Y}=0 .
$$

Из уравнения (4.9) и скачков на поверхности $S$ нормальной производной обобщенного потенциала простого слоя 


$$
V(X)=\int_{S} G_{0}(Y, X) \sigma_{0}(Y) d Y
$$

следует, что $V(X) \equiv$ const и

$$
\lim _{X \rightarrow X_{0}} \frac{\partial V(X)}{\partial n}=-\frac{\sigma_{0}\left(X_{0}\right)}{\lambda\left(X_{0}\right)}, \quad X \in \Omega_{e}, \quad X_{0} \in S .
$$

Выберем $\sigma_{0}$ так, чтобы $V(X) \equiv 1 \quad(X \in \bar{\Omega})$. По принципу максимума все точки поверхности $S$ являются точками максимума для функции $V$, регулярной на бесконечности и имеющей на $S$ постоянное значение $\left(\left.V\right|_{s} \equiv 1\right)$. Тогда по теореме Жиро $\left[{ }^{5}\right]$ будет $\left.\frac{\partial V}{\partial n}\right|_{s} \leqslant 0$. Значит, $\sigma_{0} \geqslant 0$, и необходимое и достаточное условие (4.8) выполнено. Теорема доказана.

\section{5. Замечания}

1. Для приближенного построения решения в некоторых случаях вместо главного фундаментального решения уравнения (3.4) может оказаться удобнее использовать функцию (3.11), которая является функцией Леви для оператора $\mathfrak{M}$. Однако тогда соответствующее интегральное уравнение будет содержать интегралы от неизвестной функции не только по поверхности $S$, но и по области $\Omega$.

2. Все проведенные выше рассмотрения переносятся на краевую задачу с граничным условием вида

$$
\lambda \frac{\partial u}{\partial n}=l u+f
$$

где $l$ - линейный ограниченный самосопряженный оператор в $L_{2}(S)$. При этом решение, вообще говоря, понимается в обобщенном смысле. Если же $l$ - ограниченный оператор, действующий из $C$ в $C$, то решение будет классическим.

Граничное условие вида (5.1) в случае уравнения Лапласа встречается в задаче о малых колебаниях капиллярной жидкости, для решения которой применялся метод интегральных уравнений, совершенно аналогичный рассмотренному выше (см. $\left.\left[{ }^{6}\right]\right)$.

3. Из того, что любая периодическая функция, квадратично суммируемая на отрезке $[0, T]$, единственным образом может быть представлена рядом Фурье, и из единственности решения краевых задач относительно коэффициентов Фурье (см. раздел 2) следует единственность решения задачи (A) и (с точностью до аддитивной постоянной) задачи (B).

Автор признателен Т. Томсону и Р. Мересмаа за полезное обсуждение результатов работы.

\section{Л И Т Е Р А Т У Р А}

1. Тихонов А. Н., С а м а рский А. А., Уравнения математической физики, М., «Наука», 1966.

2. Лыков А. В., Берковский Б. М., Конвекция и тепловые волны, М., «Энергия», 1974.

3. Мышки с А. Д., Т емкин Л. А., Инж.-физ. ж., 25, № 3, 518-529 (1973).

4. Т ем кин Л. А., В кн.: Исследования по теории операторов и их приложениям, Кисв, «Наук. думка», 1979, с, 107-123, 
5. Миранда К., Уравнения с частными производными эллиптического тнпа, M., Изд-во иностр. лит., 1957.

6. Т емкин Л. А., Т е м к ин а В. С., Изв. АН СССР, Мех. жидкости и газа, № 5 , $14-22(1972)$.

Научно-исследовательский и проектно-технологический институт Таллинского

Поступила в редакцию электротехнического завода им. М. Н. Калинина

24/VIII 1979

\section{TEMKIN}

\section{PARABOOLSETE DIFERENTSIAALVORRANDITE PERIOODILISTE LAHENDITE OLESEHITAMISEST}

Artiklis on esitatud meetod teist järku osatuletistega paraboolse diferentsiaalvörrandi rajaülesande lahendamiseks algtingimuse puudumisel, juhul kui perioodiliselt mõjuvad allikad paiknevad nii vaadeldava piirkonna sees kui ka selle rajal. Selline probleem kerkib elektrimasinate ja elektroonikaseadmete soojusrežiimide uurimisel. Olesanne taandub ekvivalentsete Fredholmi integraalvörrandite süsteemi lahendamisele vaadeldava piirkonna rajal. Integraalvõrrandite süsteemi on uuritud potentsiaaliteooria meetodite abil.

\section{TEMKIN}

\section{ON THE CONSTRUCTION OF PERIODIC SOLUTIONS OF THE PARABOLIC EQUATION}

A method for construction of solutions of the boundary value problem without the initial condition for the parabolic partial differential equation is proposed. It is assumed that the sources temporally act periodically. These sources are distributed inside the region where the solution of the problem is to be found as are also on the boundary of the region. Such problem arises in particular at the investigation of heat regimes in electric machines and electronic equipment. This is a variant of the problem of heat waves.

The solution is searched in the form of a trigonometrical series expansion in regard to time. The boundary value problems for a system of elliptic differential equations as to the coefficients of this series are reduced to the system of Fredholm's equations on the boundary of the region. The system of integral equations with an integrable singularity is investigated with methods of potential theory. The existence and the uniqueness of the solution of the obtained system of integral equations of the second type and its equivalence to the elliptical boundary problem is proved.

It is admissible, generally speaking, that the coefficients of the mentioned parabolic differential equation and boundary condition are variable. The problem is considered in a restricted region of $m$-dimensional Euclidean space. 